\title{
Le fratture del ginocchio
}

\author{
Gianfranco Longo ${ }^{\mathrm{a}}(\bowtie)$, Roberto Sciortino \\ Via messina 825, Catania, Italia \\ agflongo@tiscali.it
}

(c) Springer-Verlag Italia 2015

Il trattamento dei traumi del ginocchio rappresenta un'ardua sfida per tutte le Unità Operative di Ortopedia e Traumatologia, sia che esse facciano parte di centri di riferimento regionale o strutture ospedaliere operanti sul territorio.

Sono generalmente conseguenti a traumi ad alta energia, traumi sportivi o traumi a bassa energia, specie nel Grande Anziano.

Diverse soluzioni terapeutiche, grazie a svariati device, possono essere adottate in relazione al tipo di frattura, distretto anatomico interessato, condizioni cliniche locali e generali, esperienza del chirurgo. Il tutto caratterizzato dalla costante ricerca di nuove soluzioni tecnologiche, nuovi concetti biomeccanici e tribologici, nuove vie chirurgiche e tecniche di impianto, al fine di ridurre al minimo il danno chirurgico e avere un precoce ripristino delle funzionalità articolare.

Tali problematiche sono state già affrontate nel corso del XXI Convegno ASOTO dove è stata presentata l'esperienza dei chirurghi ortopedici siciliani.

Questa edizione della rivista ufficiale dell'OTODI, Lo Scalpello-OTODI Educational, si prefigge quindi di cercare di definire, sulla base dell'esperienza personale di ciascuno e sulle attuali evidenze scientifiche, gli strumenti e le metodiche da impiegare per ottenere i migliori risultati terapeutici. I diversi mezzi di sintesi ultimamente sviluppati (chiodi, placche, fissatori esterni, ecc.) presentano caratteristiche meccaniche differenti grazie all'uso di nuovi biomateriali. Ovviamente, non devono mai venir meno i principi basilari del trattamento delle fratture, quali la riduzione anatomica, la stabilizzazione primaria e la precoce ripresa funzionale, in particolare in questo distretto anatomico dove spesso c'è un coinvolgimento articolare.

Abbiamo quindi cercato di ottemperare a quelli che sono gli scopi de Lo Scalpello che "intende essere uno strumento di formazione per il giovane specialista e di aggiornamento per l'ortopedico più esperto che potranno trovare in essa non solo nozioni di base della materia, ma anche nuovi percorsi clinici, innovativi inquadramenti diagnostici e la descrizione delle più recenti metodiche di trattamento".

Un ringraziamento va, infine, a tutti coloro che hanno dedicato il loro tempo alla creazione di questo volume e che quotidianamente si impegnano con abnegazione nel fare della buona traumatologia. 